\title{
Extended ionised and clumpy gas in a normal galaxy at $z=7.1$ revealed by ALMA
}

\author{
S. Carniani ${ }^{1,2}$, R. Maiolino ${ }^{1,2}$, A. Pallottini ${ }^{1,2,3}$, L. Vallini ${ }^{4}$, L. Pentericci ${ }^{5}$, A. Ferrara ${ }^{3}$, M. Castellano ${ }^{5}$, E. Vanzella ${ }^{6}$, \\ A. Grazian ${ }^{5}$, S. Gallerani ${ }^{3}$, P. Santini ${ }^{5}$, J. Wagg ${ }^{7}$, and A. Fontana ${ }^{5}$ \\ ${ }^{1}$ Cavendish Laboratory, University of Cambridge, 19 J. J. Thomson Ave., Cambridge CB3 OHE, UK \\ e-mail: sc888@mrao.cam.ac.uk \\ 2 Kavli Institute for Cosmology, University of Cambridge, Madingley Road, Cambridge CB3 OHA, UK \\ 3 Scuola Normale Superiore, Piazza dei Cavalieri 7, 56126 Pisa, Italy \\ ${ }^{4}$ Nordita, KTH Royal Institute of Technology and Stockholm University Roslagstullsbacken 23, 10691 Stockholm, Sweden \\ 5 INAF-Osservatorio Astronomico di Roma, via Frascati 33, 00040 Monteporzio, Italy \\ 6 INAF-Bologna Astronomical Observatory, via Ranzani 1, 40127 Bologna, Italy \\ 7 Square Kilometre Array Organization, Jodrell Bank Observatory, Lower Withington, Macclesfield, Cheshire SK11 9DL, UK
}

Received 27 December 2016/ Accepted 10 April 2017

\begin{abstract}
We present new ALMA observations of the [O III] $88 \mu \mathrm{m}$ line and high angular resolution observations of the [C II] $158 \mu \mathrm{m}$ line in a normal star forming galaxy at $z=7.1$. Previous [C II] observations of this galaxy had detected [C II] emission consistent with the Ly $\alpha$ redshift but spatially slightly offset relative to the optical (UV-rest frame) emission. The new [C II] observations reveal that the [C II] emission is partly clumpy and partly diffuse on scales larger than about $1 \mathrm{kpc}$. [O III] emission is also detected at high significance, offset relative to the optical counterpart in the same direction as the [C II] clumps, but mostly not overlapping with the bulk of the $[\mathrm{C} \mathrm{II}]$ emission. The offset between different emission components (optical/UV and different far-IR tracers) is similar to that which is observed in much more powerful starbursts at high redshift. We show that the [O III] emitting clump cannot be explained in terms of diffuse gas excited by the UV radiation emitted by the optical galaxy, but it requires excitation by in-situ (slightly dust obscured) star formation, at a rate of about $7 M_{\odot} \mathrm{yr}^{-1}$. Within $20 \mathrm{kpc}$ from the optical galaxy the ALMA data reveal two additional [O III] emitting systems, which must be star forming companions. We discuss that the complex properties revealed by ALMA in the $z \sim 7.1$ galaxy are consistent with expectations by recent models and cosmological simulations, in which differential dust extinction, differential excitation and different metal enrichment levels, associated with different subsystems assembling a galaxy, are responsible for the various appearance of the system when observed with distinct tracers.
\end{abstract}

Key words. galaxies: evolution - galaxies: ISM - galaxies: high-redshift - infrared: general

\section{Introduction}

Characterising the primeval galaxies of the Universe entails the challenging goal of observing galaxies with modest star formation rates $\left(<10 M_{\odot} \mathrm{yr}^{-1}\right.$; Salvaterra et al. 2011; Finkelstein et al. 2012; Dayal et al. 2014; Robertson et al. 2015) and approaching the beginning of the reionisation epoch $(z>6)$.

To date, a large number of primeval galaxies have been identified thanks to the Lyman Break technique (e.g. McLure et al. 2013; Bradley et al. 2014; Finkelstein et al. 2015; Schmidt et al. 2014; Bouwens et al. 2015, 2016; Oesch et al. 2014, 2015; Zitrin et al. 2014; Calvi et al. 2016; Infante et al. 2015; Ishigaki et al. 2015; Kawamata et al. 2016; McLeod et al. 2015). To further our understanding on the formation and evolution of such primeval objects, we must investigate their nature and physical properties through multi-band spectroscopic observations.

In the past few years, millimetre and submillimetre spectroscopy has proven to be a powerful tool for studying the interstellar medium and kinematics of primeval galaxies through the far-infrared (FIR) atomic fine structure lines, such as [C II], [NII], [O III], redshifted to millimetre wavelengths at $z>4$. In particular, the [C II] at $158 \mu \mathrm{m}$ lines is generally the strongest FIR emission line, tracing photodissociation regions (PDRs; Madden et al. 1997; Kaufman et al. 1999; Graciá-Carpio et al. 2011; Pineda et al. 2014), neutral diffuse and partially ionised gas. This line has been detected in several quasars (QSOs), starburst, and submillimetre galaxies (SMGs) at $z>4$ (Maiolino et al. 2005, 2009, 2012; De Breuck et al. 2014; Wagg et al. 2012; Gallerani et al. 2012; Venemans et al. 2012; Carilli et al. 2013; Carniani et al. 2013; Williams et al. 2014; Riechers et al. 2014; Walter et al. 2009; Cicone et al. 2015). The high sensitivity of ALMA has recently enabled the detection of this FIR line also in faint and primeval star-forming galaxies with SFR closer to the "normal" population $\left(S F R<100 M_{\odot} \mathrm{yr}^{-1}\right)$. The properties of such galaxies in terms of [C II] emission are heterogeneous. A number of normal Lyman Break galaxies at $5<z<6$ have been detected in [C II] and it has been claimed that they follow the same [C II]-SFR relation of local galaxies (Willott et al. 2015; Capak et al. 2015). However, several [C II] detections or upper limits imply the existence of galaxies at $z \gtrsim 6$ well below the [C II]-SFR relation (Ota et al. 2014; Schaerer et al. 2015; González-López et al. 2014; Maiolino et al. 2015; Pentericci et al. 2016; Knudsen et al. 2016). For other galaxies the [C II] detection is above the local [C II]-SFR relation, including [C II] systems without optical or 
near-IR counterpart (Maiolino et al. 2015; Aravena et al. 2016; Capak et al. 2015; Knudsen et al. 2016). To make the scenario even more complex, for a significant number of galaxies at $z \sim 6-7$ the $[\mathrm{C} \mathrm{II}]$ emission appears to be significantly offset both in spatial position and in velocity. A key result in this context has been our ALMA detection of [C II] emission associated with the normal star forming galaxy $\left(S F R \approx 6 M_{\odot} \mathrm{yr}^{-1}\right) \mathrm{BDF}-3299$ at $z=7.1$ (Castellano et al. 2010; Vanzella et al. 2011). This galaxy shows [C II] emission fully consistent with the Ly $\alpha$ redshift, but spatially offset by $4 \mathrm{kpc}$ relative to the optical emission (Maiolino et al. 2015).

A promising alternative way to study the ISM properties in high- $z$ systems is given by the FIR line of oxygen [O III] at $88 \mu \mathrm{m}$. With an excitation temperature of $164 \mathrm{~K}$ and a critical density of about $510 \mathrm{~cm}^{-3}$, the [O III] is a good tracer of ionised gas in moderate density HII regions. FIR infrared observations in the local Universe up to $z \sim 0.05$ have revealed that the [O III] to FIR continuum luminosity ratio ranges between $0.03 \%$ and $2 \%$ (Malhotra et al. 2001; Negishi et al. 2001; Brauher et al. 2008) and in low-metallicity galaxies $\left(Z<1 / 3 Z_{\odot}\right)$ the oxygen line can be up $\simeq 3$ times brighter than that of [C II] (Cormier et al. 2012). Recent models have highlighted that [O III] is a key diagnostic of low metallicity ISM, especially in primeval galaxies characterised by very young stellar populations (Inoue et al. 2014; Vallini et al. 2017). In the distant Universe, the [O III] line has been detected in two gravitationally lensed AGN/starburst composite systems at redshift $z=2.8$ and $z=3.9$ (Ferkinhoff et al. 2010). The only one [O III] detection at $z>4$ has been reported by Inoue et al. (2016) who have detected the [O III] with ALMA in a star-forming galaxy at $z \sim 7.2$ and from which they estimate an oxygen abundance of about one-tenth Solar.

We have obtained new ALMA [C II] and [O III] observations of the star-forming galaxy BDF-3299 at $z=7.1$. This galaxy is located in a region characterised by a significant overdensity of LBGs at $z \sim 7$ (Castellano et al. 2016). Assuming no dust extinction, this galaxy has a $S F R=5.7 M_{\odot} \mathrm{yr}^{-1}$ (Maiolino et al. 2015) and previous ALMA observations have revealed a displaced [C II] emission relative to optical emission. The offset is not ascribed to astrometric uncertainties, as astrometry is checked through serendipitous sources. The new ALMA [C II] observations have a higher angular resolution and, along with the [O III] data, enable us to investigate more in detail the properties of the interstellar and circumgalactic medium of this primeval system.

The paper is organised as follow. Section 2 describes the ALMA observations. In Sect. 3 we analyse the continuum emission at $410 \mathrm{GHz}$ and $230 \mathrm{GHz}$. We investigate the properties of [O III] and [C II] emission in Sects. 5 and 4, respectively. In Sect. 6, we interpretate our results and we summarise the study and give our conclusions in Sect. 7.

Throughout this work we adopt the following cosmological parameter $H_{0}=67.3 \mathrm{~km} \mathrm{~s}^{-1} \mathrm{Mpc}^{-1}, \Omega_{\mathrm{M}}=0.315, \Omega_{\Lambda}=0.685$ (Planck Collaboration XVI 2014).

\section{Observations}

ALMA observations were obtained in Cycle 2 within the project 2013.1.00433.S. During this ALMA project BDF-3299 was observed both in Band $6(230 \mathrm{GHz})$ and in Band $8(410 \mathrm{GHz})$.

In Band 6 we re-observed the [C II] emission in BDF-3299 aiming at achieving the same sensitivity of previous observations obtained in Cycle 1 (Maiolino et al. 2015; Carniani et al. 2015), but with higher $(\times 8)$ spatial resolution. ALMA Band-6 observations were carried out on 2015 June 30 and July 1 with a extended configuration (longest baseline $=1570 \mathrm{~m}$ ) and a precipitable water vapour of PWV $=0.8 \mathrm{~mm}$. The on-source integration time was about $3 \mathrm{~h}$. The observations were performed in frequency division mode (FDM) with a total bandwidth of $7.5 \mathrm{GHz}$, divided in to four spectral windows (SPWs) of $1.875 \mathrm{GHz}$. One of the SPWs was centred on the [C II] redshifted frequency, which was observed with a spectral resolution of $1.95 \mathrm{MHZ}\left(\sim 2.5 \mathrm{~km} \mathrm{~s}^{-1}\right)$.

In addition to the Band 6 observations, the ALMA project aimed at mapping the [O III] at $88 \mu \mathrm{m}$, which is redshifted to 418.5 GHz at $z=7.107$. Band 8 observations were obtained on 2015 May 1 and 15. The array configuration was composed by $5512-\mathrm{m}$ antennas with baseline length in a range between $15 \mathrm{~m}$ and $560 \mathrm{~m}$. The total on-source observing time was about $73 \mathrm{~min}$ and the PWV was $0.5-0.6 \mathrm{~mm}$. The observations were carried out in FDM and the spectral band was set up to a spectral resolution of $1.95 \mathrm{MHz}\left(\sim 1.4 \mathrm{~km} \mathrm{~s}^{-1}\right)$. One of the four SPWs was centred on the expected redshifted frequency of the [O III].

The bandpass calibrator was J2258-2758 for both ALMA bands. Neptune and Ceres were observed as flux calibrators, and J1924-2914 and J2223-3137 observation were used for phase and gain calibration in the Band 8 and 6 , respectively. Unfortunately, the phase calibrator J2223-3137 observed in Band-6 suffered from instrumental errors (differential timing among the different antennae, resulting in phase shifts across the field of view), therefore it has been not suitable to calibrate and correct the variation of phase and amplitude with time. We therefore used the bandpass calibrator J2258-2758, which was observed regularly during the ALMA programme, as phase calibrator in Band 6. Since the bandpass calibrator is usually observed for time scales shorter than those of phase calibrator, the final phase errors are larger than the expected ones and the sensitivity of final images is worse than that requested. Indeed, the main effects of phase errors on interferometry are the loss of sensitivity due to decorrelation and the degradation of image quality due to scattering of flux throughout the image. In conclusion, the Cycle 2 Band-6 high resolution data have a sensitivity lower than that obtained in Cycle 1, although the on-source time and the weather conditions are similar.

Band 8 and 6 data were calibrated by using the CASA software version v4.5.3. We produced final continuum images and cubes with the routine CLEAN, selecting a natural weighting to optimise the sensitivity. Because a bright serendipitous source (flux density $S_{\text {Band-6 }}=400 \mu \mathrm{Jy}$; Maiolino et al. 2015; Carniani et al. 2015) is present in the BDF-3299 field of view, the cleaning of the continuum maps was performed with 500 iterations. No cleaning was performed in any cube, since there are no bright line emission in the field of view and the continuum flux of the serendipitous sources is not high enough to enable channel-by-channel cleaning. The achieved sensitivities and angular resolution are listed in the Table 1 . The sensitivity is about a factor 1.5 worse than achieved in Cycle 1 at lower angular resolution.

Finally, we combined Band-6 data obtained in Cycle 1 to the new one observed in Cycle 2. Before combining the visibilities of two observations, we manually re-scaled their relative weights so that they matched the noise variance measured in the two datasets. The combined data set results into a continuum sensitivity of $7 \mu \mathrm{Jy}$ and a combined angular resolution of about $0.6^{\prime \prime}$.

In this work we also make use of the Hubble Space Telescope (HST) F105W ( $Y$-band hereafter) imaging of the BDF field obtained under cycle 22 programme 13688 and described in Castellano et al. (2016). The relevant $S / N=10 \mathrm{mag}$ limit is $Y_{\mathrm{AB}}=28$ in apertures of $0.38^{\prime \prime}$, which is two times larger 
Table 1. Summary of the ALMA observations.

\begin{tabular}{lcccccc}
\hline \hline Observations & $\begin{array}{c}\text { Frequency } \\
{[\mathrm{GHz}]}\end{array}$ & $\begin{array}{c}\sigma_{\text {cont }} \\
{[\mu \mathrm{Jy} / \mathrm{beam}]}\end{array}$ & $\begin{array}{c}\sigma_{100 \mathrm{~km} \mathrm{~s}^{-1}} \\
{[\mu \mathrm{Jy} / \mathrm{beam}]}\end{array}$ & Beam & $\begin{array}{c}\text { Angular } \\
\text { resolution }\end{array}$ & $\begin{array}{c}\text { Primary } \\
\text { beam }\end{array}$ \\
\hline Band 8 Cycle 2 & 410 & 40 & 260 & $0.5^{\prime \prime} \times 0.4^{\prime \prime}$ & & $16^{\prime \prime}$ \\
\hline Band 6 Cycle 1 & 230 & 8 & 62 & $0.8^{\prime \prime} \times 0.6^{\prime \prime}$ & low & $22^{\prime \prime}$ \\
Band 6 Cycle 2 & 230 & 11 & 90 & $0.3^{\prime \prime} \times 0.2^{\prime \prime}$ & high & $22^{\prime \prime}$ \\
Band 6 Cycle 1+2 & 230 & 7 & 58 & $0.6^{\prime \prime} \times 0.5^{\prime \prime}$ & medium & $22^{\prime \prime}$ \\
\hline
\end{tabular}

than the HST angular resolution in $Y$-band $\left(0.19^{\prime \prime}\right)$. Following Maiolino et al. (2015) we matched the ALMA observations to the HST images and we applied a position shift to the $Y$-band by $\sim 0.4^{\prime \prime}$ toward the South-East, based on the consistent continuum offset measured on the two serendipitous sources.

\section{Continuum emission}

Figure 1 shows the ALMA continuum maps at $410 \mathrm{GHz}$ (Band 8) and at $230 \mathrm{GHz}$ (Band 6) around the target BDF-3299. Band-6 image is obtained combining Cycle 1 to Cycle 2 data (sensitivity and angular resolution are listed in Table 1). The FIR emission is not detected in either continuum maps at the location of BDF-3299.

At this rest-frame wavelengths the emission is due to dust thermal emission heated by the radiation field of young stars. The non detections indicate that BDF-3299 is characterised by low dust content $\left(M_{\text {dust }}<5 \times 10^{7}\right)$, as already discussed by Maiolino et al. (2015). A similar result has been claimed in recent studies of high- $z(>6)$ star-forming galaxies, in which the FIR emission relative to the UV is similar to that observed in nearby dwarf or irregular galaxies (Walter et al. 2012; Kanekar et al. 2013; Ouchi et al. 2013; González-López et al. 2014; Ota et al. 2014; Schaerer et al. 2015; Willott et al. 2015; Bouwens et al. 2016).

Assuming a dust temperature range between $25 \mathrm{~K}$ and $45 \mathrm{~K}$ (Schaerer et al. 2015), we derive a $2 \sigma$ upper limit estimates for the infrared luminosity $L_{\text {FIR }}<10^{11} L_{\odot}$. If we adopt the "standard" FIR-SFR conversion factor for local galaxies (Kennicutt \& Evans 2012) this upper limit on the FIR luminosity translates into an $2 \sigma$ upper limit on the IR-derived star formation rate of $S F R<12 M_{\odot} \mathrm{yr}^{-1}$, which is consistent with the SFR inferred for the rest-frame UV continuum $\left(5.7 M_{\odot} \mathrm{yr}^{-1}\right)$. However, since the galaxy is dust-poor, the upper limit on the FIR luminosity likely translates into a higher limit on the SFR.

\section{4. [C II] emission}

In this section we compare the maps of the carbon line obtained with different ALMA configurations: semi-compact array (low angular resolution; Maiolino et al. 2015), extended array (high angular resolution) and combined data. We recall that the previous [C II] observation of this source had an angular resolution of about $0.7^{\prime \prime}$, while the sensitivity was higher than the new high resolution data, as reported in Table 1.

We compare the spectra at the position of the [C II] detections obtained from different ALMA array configurations. The top panel of Fig. 2a shows the spectrum of the [C II] emission extracted from the dataset with high angular resolution $\left(\sim 0.3^{\prime \prime} \times 0.2^{\prime \prime}\right)$. The $[\mathrm{C} \mathrm{II}]$ is only marginally detected $(S / N \sim 2)$. This is only partly due to the lower sensitivity of the new observations. The comparison with the spectrum obtained with lower resolution observation obtained in Cycle 1 (bottom panel of
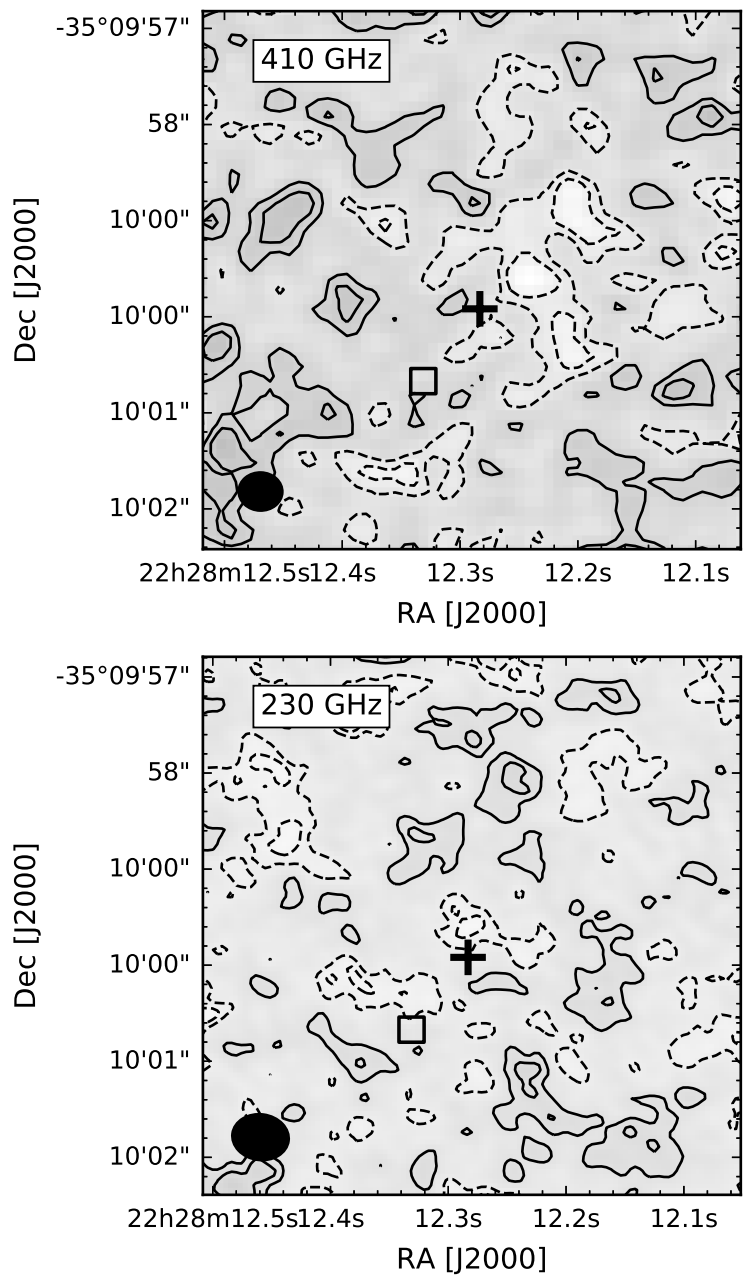

Fig. 1. ALMA continuum map in band 8 (410 GHz; top) and in Band 6 $(230 \mathrm{GHz}$, bottom $)$. Black solid contours are at levels of 1 and $2 \sigma$, where i.e $\sigma=40 \mu \mathrm{Jy} / \mathrm{beam}$ and $\sigma=7 \mu \mathrm{Jy} /$ beam in the two bands, respectively. Dashed contours trace negative levels at -1 and $-2 \sigma$. The synthesised beams are shown in the bottom left corners. The position of BDF-3299 measured in the HST $Y$-band is marked with a cross. The square mark shows the location of the [C II] detection.

Fig. 2a) confirms that the high resolution data also miss a significant fraction of the line flux, which is an indication that the total emission is not powered by a compact source with size smaller than $0.3^{\prime \prime}(\sim 1.6 \mathrm{kpc})$. The diffuse [C II] emission is resolved out in the ALMA extended configuration (see Appendix A). By measuring the line flux (Table 2) we derive that most of the total emission comes from a more extend region. We conclude that about $70 \%( \pm 20 \%)$ of the flux in the [C II] system is extended and diffuse on scales larger than about $1 \mathrm{kpc}$, which is consistent with the idea that the bulk of the [C II] emission is associated with relatively diffuse gas on scale larger than the star formign 
Table 2. Properties of the [C II] emission in BDF-3299 for the different datasets obtained with ALMA.

\begin{tabular}{lccc}
\hline \hline & $\begin{array}{c}\text { Low AR } \\
\text { dataset }\end{array}$ & $\begin{array}{c}\text { High AR } \\
\text { dataset }\end{array}$ & $\begin{array}{c}\text { Combined } \\
\text { dataset }\end{array}$ \\
\hline$\Delta V\left[\mathrm{~km} \mathrm{~s}^{-1}\right]$ & $-71 \pm 10$ & - & $-64 \pm 10$ \\
$F W H M\left[\mathrm{~km} \mathrm{~s}^{-1}\right]$ & $102 \pm 21$ & - & $75 \pm 20$ \\
Peak emission $\left[\mathrm{mJy} / \mathrm{beam} \mathrm{km} \mathrm{s}^{-1}\right]$ & $29.1 \pm 6.4$ & $3.0 \pm 1.3$ & $26.8 \pm 5.6$ \\
Flux ${ }^{\star}\left[\mathrm{mJy} \mathrm{km} \mathrm{s}^{-1}\right]$ & $49 \pm 7$ & $15 \pm 8$ & $39 \pm 6$ \\
Luminosity $\left[10^{7} L_{\odot}\right]$ & $5.9 \pm 0.8$ & $1.9 \pm 1.0$ & $4.9 \pm 0.6$ \\
\hline
\end{tabular}

Notes. ${ }^{\star}$ Following Maiolino et al. (2015) we have applied a flux aperture correction since the extraction aperture has a size close to the beam.

regions, as expected by recent model of primeval galaxies (e.g. Vallini et al. 2013, 2015; Pallottini et al. 2017; Katz et al. 2016).

By combining the visibilities of the two observations obtained in Cycle 1 and 2 we generate a single cube whose resulting angular resolution is $\sim 0.6^{\prime \prime}$ and a sensitivity of $58 \mu \mathrm{Jy} / \mathrm{beam}$ in spectral bins of $100 \mathrm{~km} \mathrm{~s}^{-1}$ (Table 1), which is slightly higher than the sensitivity reached in the two separate datasets. The [C II] spectrum obtained from the combined data is shown in the second panel of Fig. 2a. By integrating the line under the shaded gold region, we obtain a significance of the detection of $6.5^{1}$. The flux is extracted in the same aperture as the spectrum in Maiolino et al. (2015) and we applied the aperture correction estimated by using the bright serendipitous source as in Maiolino et al. (2015). We note that the integrated flux obtained from the combined observations (Table 2) is consistent with the estimates obtained by the low angular resolution data alone previously reported by Maiolino et al. (2015). The [CII] line in the combined dataset is slightly narrower $\left(F W H M=75 \pm 20 \mathrm{~km} \mathrm{~s}^{-1}\right)$ than previously reported in the lower resolution data $(F W H M=$ $102 \pm 21 \mathrm{~km} \mathrm{~s}^{-1}$ ), but still consistent within uncertainties. The map resulting from the combined set is shown in Fig. 2b, which, as expected, is clearly characterised by a more clumpy structure than the low-resolution map (shown in blue). It should be noted that the signal to noise of the peak emission $(4.8 \sigma$, Table 2$)$ is not as high as the integrated flux significance, simply because the emission is resolved and extended, hence the significance of the line detection is higher than what inferred by the flux/beam of the emission peak in the map. Additionally, the significance of the peak emission is slightly higher than what previously found in the lower resolution data alone (Table 2), which can be explained in terms of beam dilution of the latter and higher sensitivity to clumpy emission of the former.

Comparing the map and the spectra obtained with different ALMA array configuration, we conclude that the new observations confirm that the spatially-offset [C II] emission is spatially extended and reveal that such extension is due both to multiple smaller ( $\lesssim 1-2 \mathrm{kpc}$ ) clumps, distributed on such large scales, and to diffuse extended emission.

We finally note that at the location of the optical ( $Y$-band) counterpart the new combined map confirms the marginal detection $(\sim 2 \sigma)$ of some [C II] emission.

\section{5. [O III] emission}

\subsection{Line detection}

We have searched for [O III] emission in the band 8 cube, within a few $100 \mathrm{~km} \mathrm{~s}^{-1}$ of the Ly $\alpha$ emission. We detect three

\footnotetext{
1 This is the "spectral" significance, i.e. relative to the noise in the same spectrum.
}
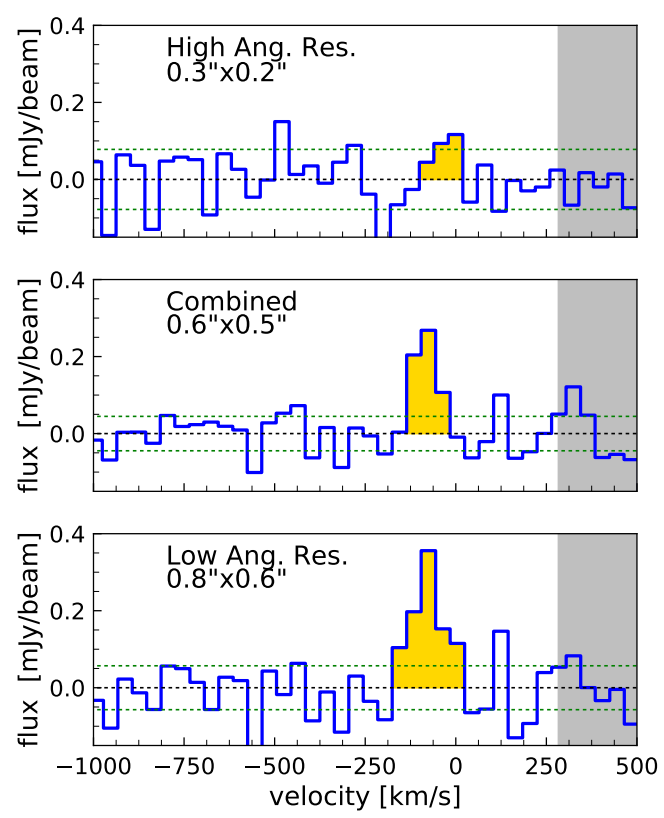

(a)

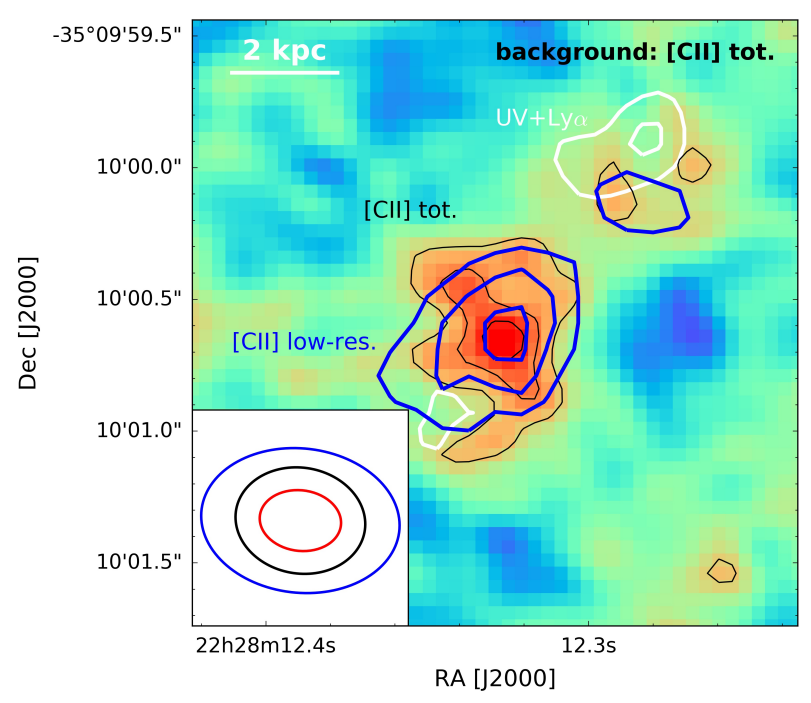

(b)

Fig. 2. Panel a) ALMA spectrum at three different angular resolution (high, medium and low) extracted at the peak position of the [C II] emission with a spectral resolution of $40 \mathrm{~km} \mathrm{~s}^{-1}$. The rms noise levels in each spectrum is shown by the green dotted line. The grey shaded region indicates the part of the spectrum affected by higher noise because of atmospheric absorption. Panel $b$ ) Map of the [C II] emission obtained by combing the two datasets (medium angular resolution) in which black contours are in steps of $1 \sigma=5.6 \mathrm{mJy} /$ beam km s$~^{-1}$, starting at $2 \sigma$. Blue contours trace the [C II] surface brightness using the low angular resolution data only (Maiolino et al. 2015) and contours are at levels 2-4 times noise per beam. The white contours trace the $Y$-band emission (UV-rest frame and $\operatorname{Ly} \alpha$ ). The bottom-left corner shows the synthesised beam of the high (red), medium (black) and low (blue) angular-resolution datasets, respectively (see Table 1).

[O III] emission line systems. On the flux map (in units of flux/beam) their emission peaks have a signal-to-noise ratio $S / N \gtrsim 5$. The integrated line emission is detected at a level of confidence $>6.5 \sigma$. The reliability of these detections, including the comparison with the "negatives" detection rate, is discussed in the Appendix. 
Table 3. Properties of the [O III] clumps observed in the field of view of BDF-3299.

\begin{tabular}{lccccccc}
\hline \hline Clump & $S / N_{\text {integrated }^{a}}$ & $S / N_{\text {peak }}{ }^{b}$ & $\begin{array}{c}F W H M \\
{\left[\mathrm{~km} \mathrm{~s}^{-1}\right]}\end{array}$ & $\begin{array}{c}v_{[\mathrm{O} \text { III }]}-v_{\text {Ly } \alpha} \\
{\left[\mathrm{km} \mathrm{s}^{-1}\right]}\end{array}$ & $\begin{array}{c}F([\mathrm{O} \text { III }]) \\
{\left[\mathrm{mJy} \mathrm{km} \mathrm{s}^{-1}\right]}\end{array}$ & $\begin{array}{c}L([\mathrm{O} \text { III }]) \\
{\left[10^{8} L_{\odot}\right]}\end{array}$ & $\begin{array}{c}\text { Distance } \\
{\left[{ }^{\prime \prime}\right]}\end{array}$ \\
\hline clump-I & 7.2 & 5.0 & $40 \pm 10$ & $440 \pm 5$ & $85 \pm 12$ & $1.8 \pm 0.2$ & 0.7 \\
clump-II & 6.9 & 5.3 & $40 \pm 10$ & $32 \pm 5$ & $100 \pm 14$ & $2.2 \pm 0.3$ & 2.5 \\
clump-III & 6.7 & 5.6 & $80 \pm 10$ & $110 \pm 10$ & $260 \pm 40$ & $5.8 \pm 0.6$ & 6.5 \\
\hline
\end{tabular}

Notes. ${ }^{(a)}$ The integrated significance is the ratio between the integrated flux of the line and the relative error calculated in the extracted spectrum. (b) Significance of the line emission "peak" in the collapsed image. The peak emission significance is slightly lower than the line integrated significance because the emission is spatially slightly extended.
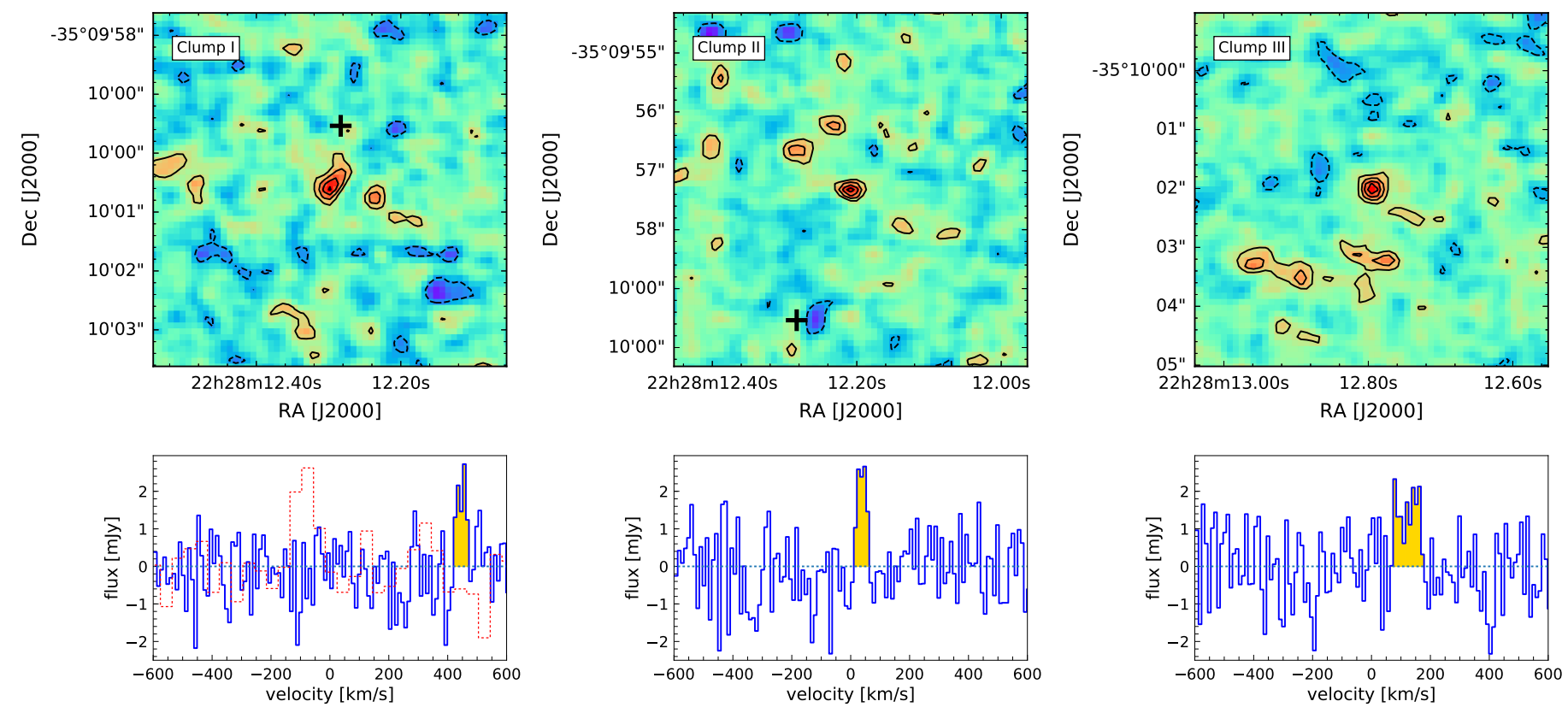

Fig. 3. The top panels show the flux maps of the three [O III] detections, clump-I, -II, and -III (from left to right). Black contours show emission at levels of 2, 3, 4 and $5 \sigma$. The location of the BDF-3299 galaxy is marked as a black cross (in the third panel the mark is outside of the field of view). The bottom panels show the spectra of the line emitters at a spectral resolution of $10 \mathrm{~km} \mathrm{~s}^{-1}$. The yellow shaded region shows the region used to integrate the flux to obtain the maps in the upper panels. Velocities are relative to the Ly $\alpha$ peak of BDF-3299. The red dotted spectrum in the left panel show the [CII] spectrum shown in Fig. 2 and scaled arbitrarily up in flux density for comparison.

The properties of the $[\mathrm{O}$ III] detections are summarised in Table 3 and the [O III] flux maps of the three detections are shown in the top panels of Fig. 3, obtained by integrating the line profile under the velocity range highlighted by the shaded region in the respective spectra (bottom panels of Fig. 3). The black cross in the maps shows the location of the optical counterpart of BDF-3299 from the HST $Y$-band image. We note that the significance of the peak emission in the maps (third column of Table 3 ) is slightly lower then the line integrated significance (second column of Table 3) because the emission is spatially slightly resolved.

The [O III] clumps are located within $\sim 6.5^{\prime \prime}(\sim 34 \mathrm{kpc})$ from the star-forming galaxy BDF-3299 and have velocities within $500 \mathrm{~km} \mathrm{~s}^{-1}$ relative to the Ly $\alpha$ peak (Table 3). One of the three detections (clump-I in Table 3) is located very close to the optical $Y$-band counterpart of BDF-3299, but it has a small offset of $0.6^{\prime \prime}$ (i.e. $3.5 \mathrm{kpc}$ ) in the same direction as the [C II] offset. However, as shown in Fig. 4, it is not coincident with [C II] either. The [O III] emission is only marginally overlapping with the [C II] emission, the two emission peaks being offset by $0.2^{\prime \prime}$ $(\sim 1 \mathrm{kpc})$, which is slightly larger than the uncertainties of $0.15^{\prime \prime}$ based on the significance of the detections and beam size.
To verify that the positional offset between the [C II] and [O III] emission is not caused by an astrometric issue, we compare the centroid of the continuum emission in Band- 6 and -8 of the brightest serendipitous source located at north-west. The source is detected in Band 8 at a level of $33 \sigma$ and its centroid position is consistent with that in Band 6 (inset in Fig. 4). This nice agreement confirms that the spatial offset between the two clumps is real. In addition, the central velocity of the [O III] is redshifted by about $440 \mathrm{~km} \mathrm{~s}^{-1}$ with respect to the Ly $\alpha$ peak and about $500 \mathrm{~km} \mathrm{~s}^{-1}$ relative to the [C II] emission. The two emission lines, [O III] and [C II], thus are tracing two different clumps. The far-infrared properties of the optical galaxy and the two clumps are summarised in the Table 4 . The spatial and spectral offsets between [O III], [C II] and Ly $\alpha$ emission will be discussed in details in Sect. 6 .

\subsection{Reliability of [O III] detections}

In this section we discuss the significance of the [O III] detections checking whether negative line emitters are detected with the same significance or not.

We performed a blind search for positive and negative line emitters within the primary beam area $\left(F W H M=16^{\prime \prime}\right)$ and 
Table 4. Summary of the far-Infrared properties at the location of the optical gaalxy, [C II] and [O III] clumps.

\begin{tabular}{lccc}
\hline \hline & Optical clump & {$[\mathrm{C}$ II] clump } & [O III] clump \\
\hline $\mathrm{S}_{230 \mathrm{GHz}}[\mu \mathrm{Jy}]$ & $<21$ & $<21$ & $<21$ \\
$\mathrm{~S}_{[\mathrm{CII}]}\left[\mathrm{mJy} \mathrm{km} \mathrm{s}^{-1}\right]$ & $<19$ & $39 \pm 6$ & $<19$ \\
$L_{[\mathrm{CII}]}\left[10^{7} L_{\odot}\right]$ & $<2$ & $4.9 \pm 0.6$ & $<2$ \\
$S_{\text {[OIII] }}\left[\mathrm{mJy} \mathrm{km} \mathrm{s}^{-1}\right]$ & $<30$ & $<30$ & $85 \pm 12$ \\
$L_{\text {[OIII] }]}\left[10^{8} L_{\odot}\right]$ & $<5.5$ & $<5.5$ & $1.8 \pm 0.2$ \\
\hline
\end{tabular}

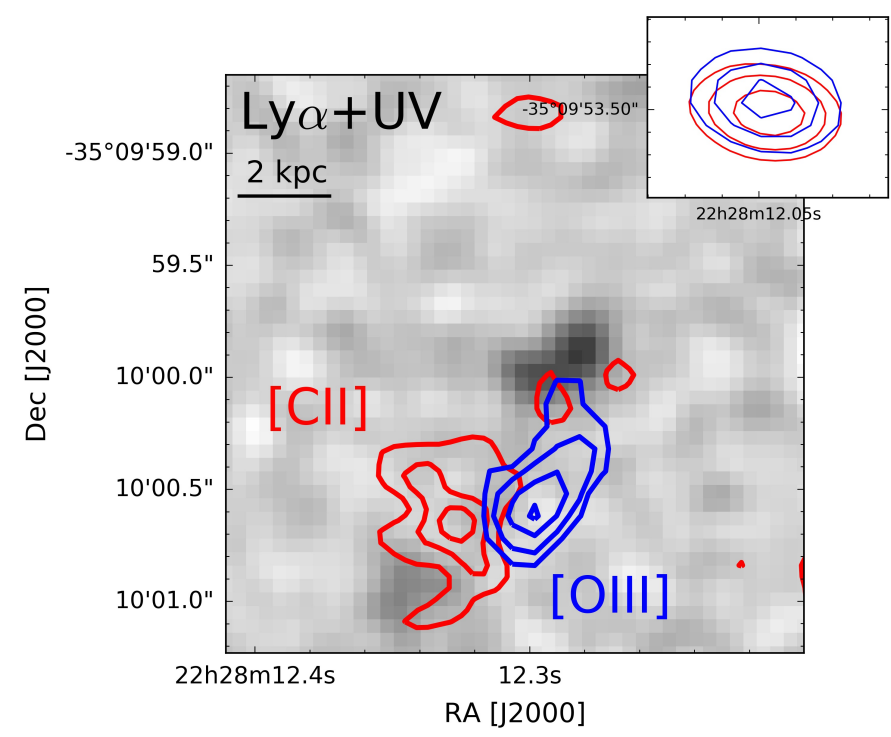

Fig. 4. The HST $Y$-band image of the BDF-3299 field is shown in the background in grey scale. The blue contours show the [O III] map of clump-I. The red contours show the [C II] emission. In both maps the contours are at levels of 2,3 , and $4 \sigma$. Since the emission is resolved in both cases, the peak emission is not representative of the significance of the emission when extracted from the entire emission region. The inset shows in blue and red contours the continuum emission of the bright serendipitous source at North-West in Band- 6 and -8 , respectively, showing that the astrometry in Band- 8 is consistent within the error $\left(0.1^{\prime \prime}\right)$ with the astrometry in Band-6.

within a velocity range $|v|<500 \mathrm{~km} \mathrm{~s}^{-1}$ relative to the redshift of the Ly $\alpha$ peak. We excluded the region within $2^{\prime \prime}$ from the continuum serendipitous source, since the uncleaned continuum map shows some low-level sidelobe residuals in this area. In the defined velocity range, our blind line search algorithm scans the ALMA datacube rebinning it in different velocity channels, from $30 \mathrm{~km} \mathrm{~s}^{-1}$ to $300 \mathrm{~km} \mathrm{~s}^{-1}$, in step of $15 \mathrm{~km} \mathrm{~s}^{-1}$. In each averaged velocity channel plans we computed the rms and we searched for positive (negative) peaks exceeding a $S / N=4(-4)$. Top panel of Fig. 5 shows the distribution of positive (red) and negative (blue) detections as a function of $\mathrm{S} / \mathrm{N}$ of the "peak" in each map. At $S / N \gtrsim 5$, there are six potential positive detections and only one negative. Since the positive ones could be spurious because of noise fluctuations, we compare this distribution to that expected in blank fields. We simulated 100 blank datacubes similar to the real data and we applied on them the same source extraction algorithm used in the ALMA observations. In the simulated data, the distribution of positive peaks is similar to that of negative ones and we expect to have only one negative and positive detections per datacube at $S / N \sim 5$. This simulated noise distribution is shown with the dotted histogram in the top panel of Fig. 5.
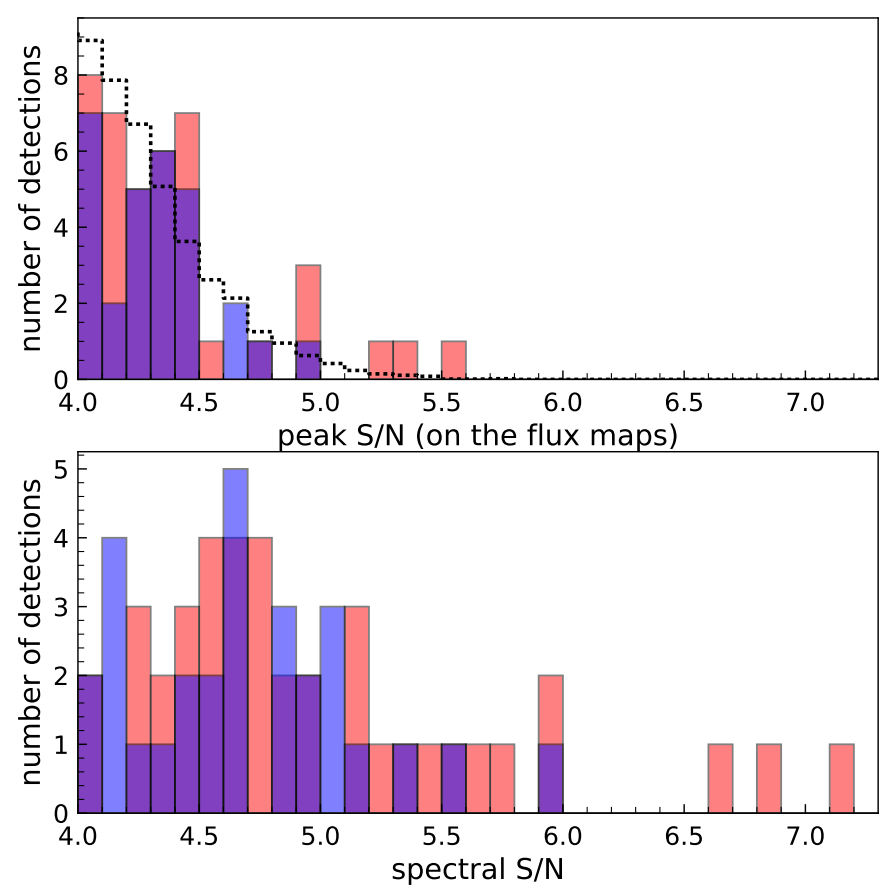

Fig. 5. Top: number of positive (red) and negative (blue) detections as a function of $\mathrm{S} / \mathrm{N}$, which is defined as the ratio of the peak emission to the noise in the flux map. The dotted black line shows the distribution of positive (or negative) detections obtained from 100 pure noise datacubes and normalised to one datacube. Bottom: number of positive (red) and negative (blue) detections as a function of "spectral" significance, i.e. relative to the noise in the same spectrum, which is defined as the ratio of the flux line to the relative error.

We note that recent similar blind search works claim a reliability level of $50 \%$ at $S / N=5$ (Walter et al. 2016; Aravena et al. 2016), so half of our detections at $S / N \geq 5$ could be spurious. For that reason, we performed a further analysis. For each putative detections (positive and negative) we extracted the relative spectrum and we estimated the flux line and relative error in the extracted spectrum. We note that the negative line emitters have a line flux with a level of confidence below $6 \sigma$ (bottom panel of Fig. 5) and only three out of six positive detections with $S / N \gtrsim 5$ in the previous analysis have level of confidence $>6$ as expected from the reliability level. This support the reliability of the three selected putative positive detections $>6.5 \sigma$.

\section{Discussion}

\subsection{Spatial and spectral offsets}

The offset between [C II] and [O III] emission, and also relative to the optical counterpart, may appear anomalous. However, such offsets (either spatial, in velocity, or both) are also seen in some high- $z$ SMG galaxies (Riechers et al. 2014; Decarli et al. 2014; Pavesi et al. 2016). Recent ALMA observations have revealed spatial and spectral offsets between FIR-lines, such as [C II] and [O III], and optical emission (Willott et al. 2015; Capak et al. 2015; Inoue et al. 2016; Knudsen et al. 2016) also in normal high-redshift galaxies $(z>5)$, with modest SFR $\left(<100 M_{\odot} \mathrm{yr}^{-1}\right)$. Therefore, BDF-3299 may not be a rare case. These offsets have been only marginally discussed (or completely ignored) in the literature, but they are probably revealing important physical properties of these high redshift systems. 


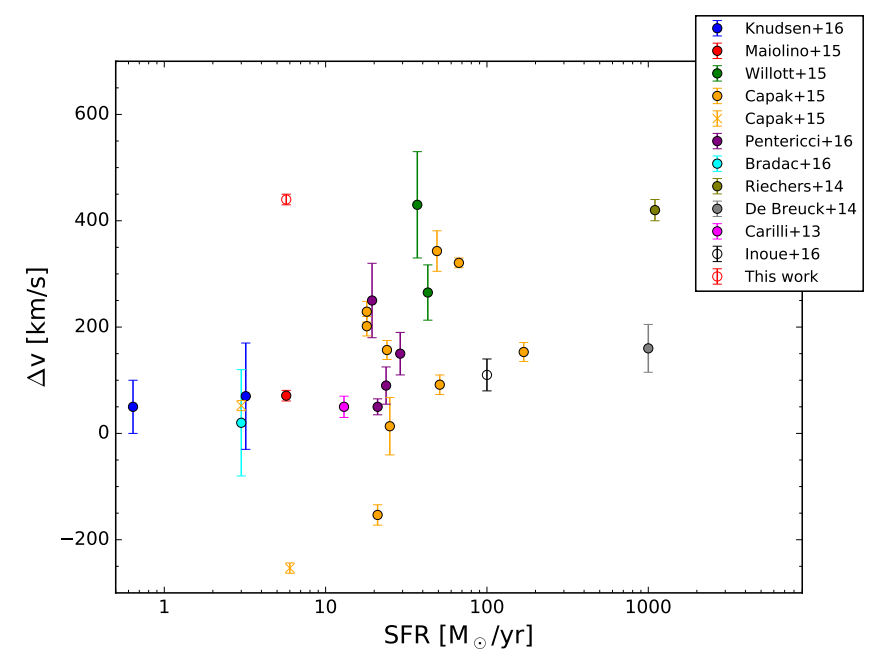

(a)

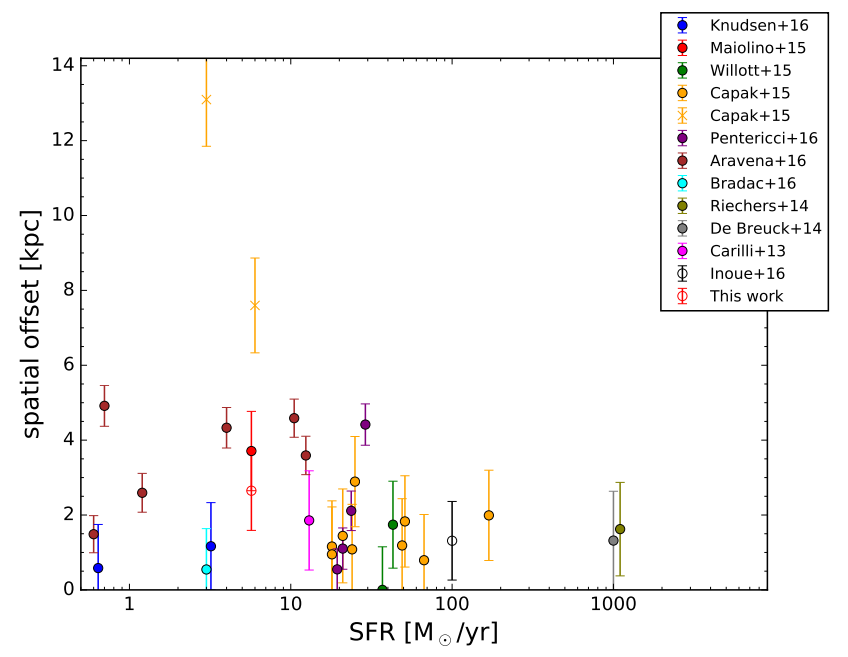

(b)

Fig. 6. Spectral $(a)$ and spatial $(b)$ offsets between the far-IR fine structure lines and the optical/UV tracers in high redshift galaxies galaxies, from the literature. The filled circles are estimated from [C II] emission obtained from Maiolino et al. (2015), Willott et al. (2015), Capak et al. (2015), Knudsen et al. (2016), Pentericci et al. (2016), Aravena et al. (2016), while the open ones denote the spectral and spatial offsets revealed from [O III] detections (this work and Inoue et al. 2016). The orange cross symbols indicates the offsets of two sources (HZ5a and HZ8W; Capak et al. 2015) for which the association between [C II] and the optical galaxy is uncertain.

In this section we make a brief statistical assessment of the offset between far-IR lines and optical counterparts in normal galaxies at $z>4$ reported in the literature.

In Fig. 6 we summarise spectral and spatial offset from literature studies observing SMG $\left(S F R>1000 M_{\odot} \mathrm{yr}^{-1}\right) z>$ 4 (Riechers et al. 2014; De Breuck et al. 2014) and primeval galaxies with $S F R<100 M_{\odot} \mathrm{yr}^{-1}$ and $z>4$ (Mallery et al. 2012; Carilli et al. 2013; Williams et al. 2014; Maiolino et al. 2015; Willott et al. 2015; Capak et al. 2015; Knudsen et al. 2016, 2017; Pentericci et al. 2016; Inoue et al. 2016; Bradač et al. 2017). Since this work does not focus on determining a correlation between the offsets and the galaxy properties, we also report the positional offset revealed in in high- $z$ galaxies whose redshifts have not been spectroscopically confirmed in the optical yet (Aravena et al. 2016). The figure is composed of panels showing the spatial and spectral offsets as a function of SFR. Spatial and spectral offsets are present in most of these sources. However no clear correlation is visible between offsets and galaxy properties. It should be noted that, by analysing the first deep ALMA continuum image of the Hubble Ultra Deep Field (HUDF), Dunlop et al. (2017) claim that, even after correcting for a systemic astrometric offset between the ALMA and HST astrometry, the HST images have residual random positional errors with a $\sigma=0.2^{\prime \prime}$, which they claim can account for offsets up to $\sim 0.5-0.6^{\prime \prime}$ (which is somewhat surprising given the small size of the area and given that this is probably the deep field studied in greatest detail). However, position offset larger than $\sim 0.5-0.6^{\prime \prime}$ cannot be associated to positional noise errors. In some studies the astrometry has been verified by using additional external datasets (e.g. Willott et al. 2015), while in our previous works we have verified the astrometry accuracy by using serendipitous sources in the field of view. Finally, a number of [C II]-emitters are simply double (with one of the two sources having no optical counterparts), clearly indicating that the absence of optical counterpart, cannot be ascribed to instrumental offsets (Capak et al. 2015). Large positional offsets between ALMA and HUDF optical and NIR observations have been also reported by Aravena et al. (2016), who found twelve
[C II] candidates with significance $>4.8 \sigma$ located at a distance up to $1^{\prime \prime}$ from the optical counterpart.

In addition to positional offsets, Fig. 6 indicates that there are velocity offsets between the FIR lines and the optical/UV spectral features, spanning a range between $-300 \mathrm{~km} \mathrm{~s}^{-1}$ and $+420 \mathrm{~km} \mathrm{~s}^{-1}$. Velocity shifts between optical/UV lines, and in particular relative to $\operatorname{Ly} \alpha$, have been found and investigated by several authors (e.g. Pettini et al. 2002; Shapley et al. 2003; Gallerani et al. 2016; Stark et al. 2017; Williams et al. 2014; Erb et al. 2014). In the case of Ly $\alpha$ IGM absorption can cause the centroid of $\operatorname{Ly} \alpha$ to be artificially redshifted (i.e. other lines blueshifted relative to Ly $\alpha$ ) up to about $100-200 \mathrm{~km} \mathrm{~s}^{-1}$; therefore, blueshifted velocities of [C II] line up to $\Delta v=v_{[\mathrm{CII}]}-v_{\mathrm{Ly} \alpha}=$ $-200 \mathrm{~km} \mathrm{~s}^{-1}$ are fully consistent with the rest-frame of the galaxy. Positive velocity shifts and velocity shifts larger than $300 \mathrm{~km} \mathrm{~s}^{-1}$ can be explained invoking outflows, often traced by $\operatorname{Ly} \alpha$ and high ionisation lines, and higher column density of neutral hydrogen in the galactic or circumgalactic medium. However, IGM absorption and outflows cannot account for velocity shifts relative to other UV transitions (e.g. CIII or UV absorption features, as in the case of Capak et al. 2015) and obviously cannot account for velocity shifts between different farIR transitions, as observed by us and others (Pavesi et al. 2016; Decarli et al. 2014). In fact, all far-IR and UV emission lines, whose emission is not absorbed by IGM, should have the same velocity offsets if they are emitted from the same region of the galaxy.

The lack of any clear correlation in Fig. 6 with the SFR and the spatial offsets larger then few kpc may yield to a further interpretation. Major merging, minor merging (i.e. accretion phases) and/or virial motions of different galaxy components in the early phases of formation, subject to different excitation mechanisms, different chemical abundances and also differential extinction effects, are likely to play a major role in the observability of different spectroscopic tracers and on their distribution and offsets. This will be discussed further in the next section.

Summarising, while some of the spatial offsets may be ascribed to positional uncertainty and some of the spectral offsets 
may be ascribed to Ly $\alpha$ IGM absorption and/or outflows, a fraction of the offsets observed between optical emission and far-IR emission (and also between different far-IR tracers) may be due to physically distinct regions of a galaxy, characterised by different physical conditions, or accretion events (minor merging) or major merging.

\subsection{Theoretical scenarios and numerical simulations}

Before discussing in detail our ALMA results on BDF-3299, in this section we briefly review the numerical simulations that have been developed in the past few years specifically focussed on the distribution of far-IR lines emission in primeval galaxies, so that these provide possible scenarios in which our data can be interpreted.

Vallini et al. (2013) take advantage of cosmological SPH simulations, combined with a radiative transfer code, to investigate the distribution of the various phases of the ISM and CGM in a primeval galaxy at $z \sim 6.6$. They predict that the bulk of the galaxy is mostly photoionised by the strong UV radiation field of young stars, and they expected tracers of ionised gas (including $[\mathrm{NII}])$ to be co-located with the regions of star formation. In their simulation [C II] is expected to survive only in (accreting) clumps of pre-enriched gas in the circumgalactic medium; in these clumps the [C II] emission results as a consequence of excitation by the UV radiation received externally from the central galaxy (i.e. not as a consequence of in situ excitation by insitu star formation). In this scenario, offset of [C II] emission relative the UV counterpart (star forming galaxy) results naturally. However, [O III] should be colocated with the UV counterpart.

Vallini et al. (2015) and Pallottini et al. (2017) expand further the model by including (i) the [C II] emission from dense PDR surrounding molecular clouds; (ii) a proper modelling of the metallicity profile in the simulated galaxy (Pallottini et al. 2015) and; (iii) by taking into account the excitation by the CMB. In these simulations, although circumgalactic [C II] is present, the peak of the [C II] emission is coincident with the location of molecular clouds, where stars form. In this scenario, although a component on large scales is present, the [C II] emission peaks are expected to trace regions of star formation, which should be coincident with the $Y$-band emission, unless dust obscuration plays a role. Vallini et al. (2017) suggests that an offset between [C II] emission and UV-stellar emission could result from a strong feedback effect, which could clean the most vigorous star forming regions, or even the entire galaxy, from the bulk of the molecular gas. Low metallicity and chemical inhomogeneity are also invoked as possible causes of the faintness of the [C II] emission; indeed, metallicity effects were already proposed in Vallini et al. (2015) to be one of the major cause of the deficit of the [C II]-SFR relation observed at high-redshift relative to the local one (e.g. De Looze et al. 2014). Vallini et al. (2017) also point out that the relative intensity of the [O III] and [C II] emission strongly depends on the intensity of the radiation field $G_{0}$, on the gas metallicity and on the evolutionary stage of the system.

Katz et al. (2016) present six cosmological hydrodynamic simulations, including radiative transfer, and investigate the evolution of star formation, chemical enrichment and the strength and hardness of the radiation field, as well as the distribution of the various gas phases (and in particular the species responsible for the emission at far-IR wavelengths) on different galactic and circumgalactic scales. They find that [C II] has a diffuse component, but its most prominent peaks are associated with dense clumps in which star formation is taking place. [O III] traces gas ionised in star forming regions, but predominantly traces those regions in which the ionisation parameter is highest. They do observe offsets between [C II] and [O III], primarily consequence of variations of the ionisation parameter. According to their simulations regions of star formation are always associated with either [C II] clumps or [O III] clumps. They suggest that offsets of the $[\mathrm{C} \mathrm{II}]$ and/or [O III] emission relative to the UV emission are primarily associated with dust extinction and/or metallicity effects. In particular, they suggest that the absence of UV counterparts in regions emitting [C II] and/or [O III] is a consequence of dust extinction. Instead, the weakness or absence of [C II] or [O III] emission in some star forming regions visibile at $\mathrm{UV}$ wavelengths is a consequence of low metallicity; more specifically, these could be low metallicity systems recently accreted through the cosmic streams, whose low dust content makes them visibile in the UV.

In the following we discuss and interpret the data specific of BDF-3299, also at the light of the scenarios discussed above.

\subsection{The lack of far-IR emission at the UV location of BDF-3299}

Although some marginal [C II] emission is detected at the location of the $Y$-band counterpart (tracing the UV rest-frame light) of BDF-3299, and although the $2 \sigma$ emissivity contour of [O III] does overlap with the $Y$-band image, both far-IR tracers peak at a few kpc away from the UV emission.

Since both [C II] and [O III] are only marginally detected at the location of the $Y$-band counterpart, we assume that neither of them is detected and we infer their upper limit. The upper limit on [C II] at the location of the UV emission was already inferred by Maiolino et al. (2015) and estimated to be $L_{[\mathrm{C} \mathrm{II}]}<2 \times 10^{7} L_{\odot}$. To estimate the upper limit on [O III] and to be consistent with the upper limit on [C II] luminosity inferred by Maiolino et al. (2015), we assume a line width of $100 \mathrm{~km} \mathrm{~s}^{-1}$; by doing so we obtain an upper limit on the [O III] luminosity of $L_{[\mathrm{O} \text { III }]}<5.5 \times$ $10^{7} L_{\odot}$.

As mentioned above, the lack of [O III] and [C II] emission at the location of the UV-emission tracing young stars, could be associated with low metallicity in the primeval galaxy. Using the CLOUDY code, Inoue et al. (2014) predict the FIR-line emissivity in HII regions from high- $z$ galaxies as a function of metallicity $(Z)$. They found that the [O III] emissivity is proportional to the metallicity at $Z<Z_{\odot}$, while the dependence becomes shallow at $Z>Z_{\odot}$. The model predicts that a galaxy with an [O III] luminosity of $5.5 \times 10^{7} L_{\odot}$ and a $S F R=7 M_{\odot} \mathrm{yr}^{-1}$ (inferred by the UV emission) can be explained with a metallicity in the range of $Z=0.1-0.3 Z_{\odot}$, depending on ionisation parameter $\left(-3.0<\log _{10} U<-1.0\right)$ and on the hydrogen number density $\left(0.0<\log _{10}\left(n_{\mathrm{H}} / \mathrm{cm}^{-3}\right)<3.0\right)$. Hence, according to these models, a metallicity lower than $Z<0.1 Z_{\odot}$ should result into an [O III] luminosity undetectable by our observations. Such low metallicity would be consistent with the low dust content discussed in Maiolino et al. (2015).

An alternative scenario, already mentioned in Maiolino et al. (2015) and in Vallini et al. (2015, 2017), is that we are observing BDF-3299 at the end of a strong feedback phase that swept away the dense gas out of the galaxy, hence suppressing the emission of far-IR lines. Ly $\alpha$ would still be detectable, despite the low gas content; indeed, Ly $\alpha$ has little dependence on the total amount of gas since it is mostly sensitive to the UV ionising flux and to the escape fraction, which might be enanched during a strong feedback event. This scenario is discussed in Vallini et al. (2013, 2015, 2017) and Olsen et al. (2015). 


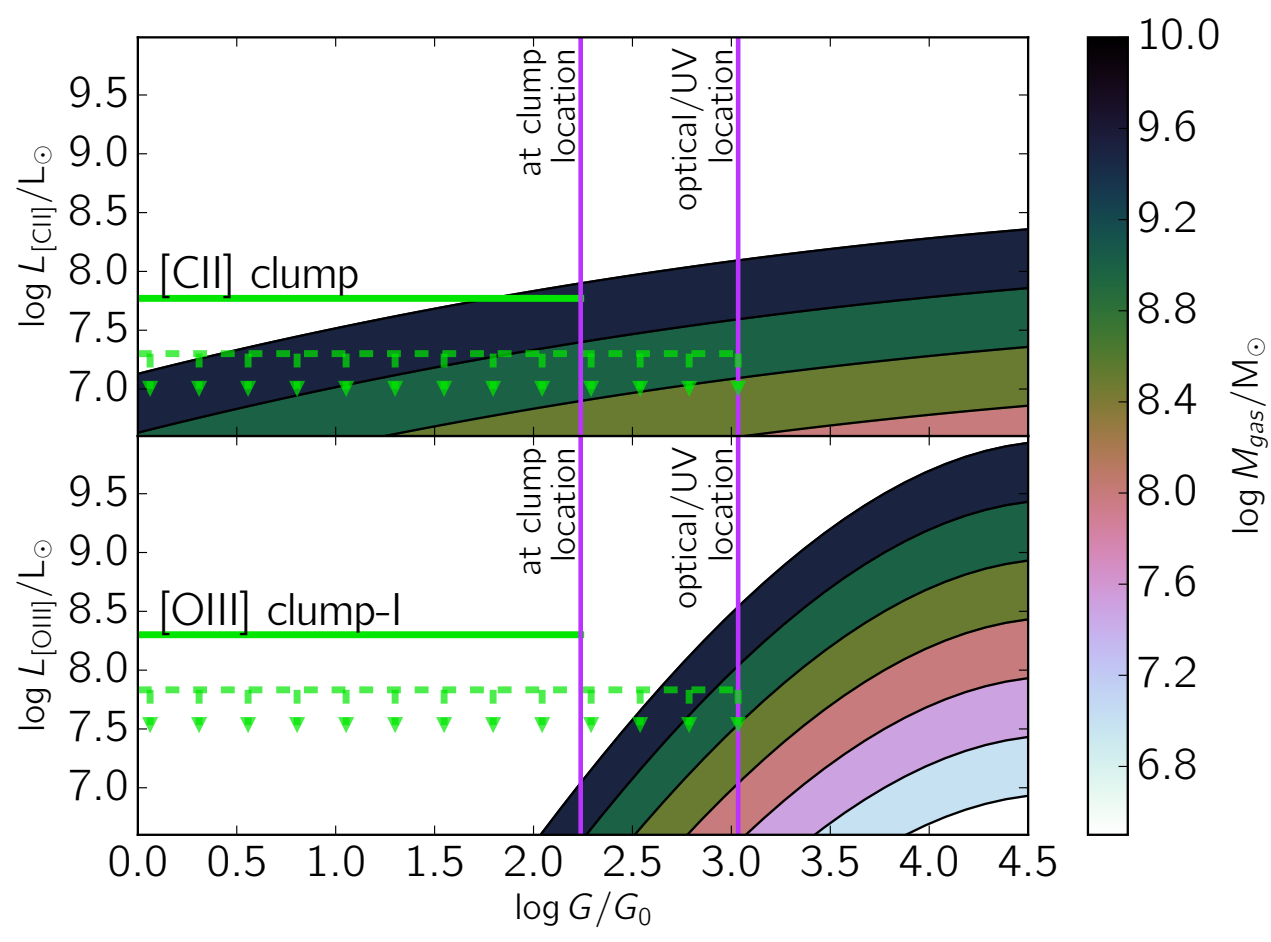

Fig. 7. Predicted [C II] (upper panel) and [O III] (lower panel) luminosities $(L)$ as a function of the incident flux $(G)$ exciting the line, inferred by using the model presented in V16, in which the colour shading is done according to $M_{\mathrm{gas}}$, as encoded in the colourbar. In each panel we plot with a horizontal green line the luminosity inferred from our observations of the [C II] clump and of the [O III] clump-I. The horizontal dashed green lines show the upper limits at the location of the $Y$-band image of BDF-3299. Additionally, we show the flux $G$ expected from BDF-3299 at the location of the [C II] and [O III] clumps and also the estimated average $G$ within the galaxy BDF-3299. See the text for details.

By using the model by Vallini et al. (2017, V16 hereafter), we can quantify the maximum gas mass contained in BDF-3299 that can be consistent with the lack of detection. V16 model the FIR emission from molecular clouds (MC) immersed in the radiation field of a parent galaxy. The model assumes that no star formation activity is present inside the clouds and accounts for photoevaporation effect due to external radiation. In V16 the MC internal structure is accounted for by considering the coexistence of different density phases $\left(10<n_{\mathrm{H}} / \mathrm{cm}^{-3}<10^{5}\right)$, that are due to competition between turbulence support and gravitational collapse (e.g. Federrath \& Klessen 2013). For the present work, we treat the FIR emitting gas as collection of MCs with total mass $M_{\text {gas }}$ and, as a baseline, we assume a gas metallicity $Z=0.2 Z_{\odot}$ (consistent with the predictions by Inoue et al. 2014). In Fig. 7 we plot the predicted FIR emissions of [C II] and [O III] as a function of incident flux $(G)$ for different $M_{\text {gas }}$. The FUV density field in BDF-3299 can be estimated by using STARBURST99 (Leitherer et al. 1999). Based on the UV photometric constraints (Vanzella et al. 2011), the galaxy has a luminosity in the FUV band of $\sim 3 \times 10^{43} \mathrm{erg} \mathrm{s}^{-1}$. To derive the size of the star-forming galaxy, we fit a two dimensional Gaussian to the $Y$-band and we estimate a size of $\sim 1 \mathrm{kpc}$ for BDF-3299, which implies a mean radiation field in the galaxy of $\sim 10^{3} G_{0}$ $\left(G_{0}=1.6 \times 10^{-3} \mathrm{erg} \mathrm{cm}^{-2} \mathrm{~s}^{-1}\right.$, Habing 1968). This value is plotted as the rightmost vertical line in Fig. 7 . We note that such a value must be taken only as an average reference, as inside a galaxy the FUV flux should be calculated accounting for the distribution of stars and gas (Wolfire et al. 2003).

The upper limits on $L_{[\mathrm{CII}]}$ an $L_{[\mathrm{O} \text { III] }}$ inferred for BDF-3299 are marked with horizontal green dashed lines. Since the [C II] and the [O III] emission are not detected at the location of the UV-emission, we conclude that the gas mass in BDF-3299 is less than $10^{9} M_{\odot}$. Of course, a different metallicity would imply a different upper limit on the gas mass (approximately scaling with the metallicity, for example see Eq. (8) in Pallottini et al. 2017).

We also note that BDF-3299 is not detected in the continuum emission at $\lambda_{\text {rest }}=88 \mu \mathrm{m}$. If a standard SED of a starburst galaxy (M82) is adapted to the observed upper limit on the continuum $\left(F_{v}<40 \mu \mathrm{Jy}\right)$ then the inferred $(2 \sigma)$ upper limit on the SFR would be $12 M_{\odot} \mathrm{yr}^{-1}$, consistent with the SFR inferred from the UV light. However, if the dust content is much lower than in local starburst templates, as a consequence of low metallicity and because of feedback removal of the ISM, then even less radiation is expected to be reprocessed in the far-IR and the constraint on the SFR would be even looser.

\subsection{The nature of the $[\mathrm{CII}]$ and [O III] emitting regions}

Two main scenarios can be envisaged for explaining the offset [C II] and [O III] emission relative to the UV emitting region. One is that, as mentioned above, these are accreting satellite clumps or clumps resulting from past outflow, and which are being excited by the UV radiation emitted by BDF-3299. The second scenario is that the line emission in these clumps is due excitation by in-situ star formation that is dust-obscured, hence not detected at UV-rest frame wavelengths. In the latter case these star forming regions could be part of the same large galaxy, but subject to differential dust extinction and differential excitation and enrichment effects, or could be galaxies in the process of (major) merging and/or accreting small galaxies (minor merging).

These scenarios can be investigated by using the same V16 models discussed above. To investigate the scenario of 
clouds being excited by the UV radiation emitted by BDF-3299 we estimate the UV flux impinging onto the [O III] and [C II] clumps by using the observed projected distance. For sake of simplicity and clarity, we assume an equal distance of $\simeq 3.5 \mathrm{kpc}$ for both the [O III] and [C II] clumps with respect to BDF3299. Hence, we obtain an intensity of the UV radiation field of $\sim 150 G_{0}$. We note that the latter is an upper limit, since if projection or absorption effects are in place then the resulting UV flux from BDF-3299 would be reduced consequently. The inferred Habing field is indicated by the leftmost vertical line in Fig. 7. The $[\mathrm{C}$ II $]$ and $[\mathrm{O} \mathrm{III}]$ observed luminosities are indicated with horizontal lines.

\subsubsection{Oxygen emission}

Clearly the observed [O III] luminosity cannot be explained in terms of external excitation from the UV radiation emitted by BDF-3299 as the implied gas mass would have to be much larger than $10^{10} M_{\odot}$. Therefore, the only viable explanation is that the [O III] is powered by in situ star formation. This is consistent with the analysis of V16, where it is shown that the typical current ALMA sensitivity is not sufficient to detect [O III] emission in clumps located a few kpc from the main galaxy with a radiation field similar to that produced by only BDF-3299. Therefore, the detection in the clump-I indicates that [O III] excitation primarily originates from in-situ star formation.

If we take the relation between SFR and [O III] luminosity for metal poor galaxies found by De Looze et al. (2014), then the [O III] luminosity observed in Clump-I yields a SFR of about $7.5 M_{\odot} \mathrm{yr}^{-1}$.

If unobscured, this should result in about the same $Y$-band flux as BDF-3299. However, at the location of Clump-I there is no $Y$-band detection, with an upper limit on the flux that is about seven times lower than the flux observed in BDF-3299. The tension can be reconciled by assuming a mild amount of dust extinction. As a matter of fact, at the rest-frame wavelength corresponding to the $Y$-band image (i.e. $\sim 1300 \AA$ ) and assuming a Calzetti attenuation curve (Calzetti et al. 2000), a reddening of only $E(B-V)=0.2$ (i.e. $A_{V} \sim 0.8$ ) would be enough to make the UV emission undetectable. In fact, with such extinction we predict a magnitude in $Y$-band $>30$, while the magnitude limit of the current HST observations is 28 at $10 \sigma)$. We note that a similar reddening value $(\langle E(B-V)\rangle \sim 0.19)$ has been recently observed in $z \sim 1$ low-metallicity $\left(Z<0.6 Z_{\odot}\right)$ star-forming galaxies (Amorín et al. 2015).

We shall also mention that the SFR of about $7.5 M_{\odot} \mathrm{yr}^{-1}$ inferred from the [O III] luminosity is compliant with the nondetection of continuum dust emission. In fact, the flux emission at $230 \mathrm{GHz}$ expected by a high- $z$ star-forming galaxy with $S F R=7.5 M_{\odot} \mathrm{yr}^{-1}$ is $\sim 5 \mu \mathrm{Jy}$ (even fainter in the low-metallicity scenario) that is below our current sensitivity. Additionally, if the in-situ star forming gas in the clump is very concentrated, then the $G$ flux would be even higher, and lower values of gas mass (and consequently SFR) are needed in order to explain the [O III] emission, as shown in Fig. 6.

We also note that the non-detection of [C II] at the location of clump-I implies $\left.L_{[\mathrm{O}} \mathrm{III}\right] / L_{[\mathrm{C} \mathrm{II}]}>8$, which is is consistent with the value expected for low metallicity galaxies and with the $[\mathrm{O}$ III $] /[\mathrm{C} \mathrm{II}]$ ratio observed by Inoue et al. (2016) in a galaxy at $z=7.2$.

The high value of the $[\mathrm{O} \mathrm{III}] /[\mathrm{C} \mathrm{II}]$ ratio can also be explained in terms of high ionisation parameter, as expected by Katz et al. (2016).
It should be noted that also the other two [O III] detections (clumps II and III), which are even farther away from BDF3299, can only be explained in terms of in-situ star formation (with star formation rates of about $10-20 M_{\odot} \mathrm{yr}^{-1}$ ). Also for these cases the lack of $Y$-band counterpart can be accounted for in terms of some dust reddening corresponding to $E(B-V)$ less than 0.3 , which is consistent, within the errors, to the dust reddening estimates reported by Amorín et al. (2015) who observed $z \sim 1$ low-metallicity $\left(Z<0.6 Z_{\odot}\right)$ star-forming galaxies with $0.1 M_{\odot} \mathrm{yr}^{-1}<S F R<35 M_{\odot} \mathrm{yr}^{-1}$.

\subsubsection{Carbon emission}

Regarding the [C II] emission clump, Fig. 7 illustrates that a satellite system with total mass of about $4 \times 10^{9} M_{\odot}$ would radiate the observed [C II] if excited externally by the UV light emitted by BDF-3299. Of course, the limiting mass depends significantly on the assumed metallicity: a metallicity higher than assumed in Fig. 7 (i.e. $Z>0.2 Z_{\odot}$ ), would relax the gas mass requirements; higher metallicity could result from pre-enrichment by past outflows from BDF-3299 or from some enrichment due to past minor star formation in the cloud, as discussed in Maiolino et al. (2015). In summary, the possibility of a satellite clump (either in the process of accreting, or resulting from previous outflow) whose [C II] emission is excited by the UV radiation emitted by BDF-3299 is viable.

The alternative scenario of excitation by in-situ star formation cannot be excluded either. Indeed, according to the [C II]-SFR correlation for low metallicity galaxies inferred by De Looze et al. (2014), the observed [C II] luminosity would imply a SFR of only $3 M_{\odot} \mathrm{yr}^{-1}$. The latter would be only marginally detected in the $Y$-band $(<3 \sigma)$ and a reddening of only $E(B-V)=$ 0.1 (i.e. $A_{V} \sim 0.4$ ) would be certainly enough to make it completely undetectable.

In the latter scenario the absence of [O III] emission can be interpreted in terms of metallicity (higher metallicity associated with the [C II] clump than in the [O III] clump), or in terms of lower ionisation parameter than in the [O III] clump, as suggested by Katz et al. (2016). The latter effect has already been observed in some SMGs at high-z (e.g. Pavesi et al. 2016; Rawle et al. 2014; Riechers et al. 2014) in which different far-IR lines show significant spatial and velocity offsets. Our result may imply that a similar scenario applies also to normal galaxies at high redshift.

To further discriminate these different scenarios higher sensitivity [C II] and [O III] ALMA data are required, possibly also including [N II] $122 \mu \mathrm{m}$, which would enable to better disentangle excitation and metallicity gradients. JWST will also greatly help by targeting the nebular lines and stellar continuum with much higher sensitivity than currently feasible and at optical rest-frame wavelengths, at which dust absorption is much reduced relative to the optical.

\section{Summary}

We have reported new ALMA millimetre observations aimed at investigating the [C $\mathrm{II}]$ at $158 \mu \mathrm{m}$ and the [O III] at $88 \mu \mathrm{m}$ emission in the star-forming $\left(S F R=5.7 M_{\odot} \mathrm{yr}^{-1}\right)$ galaxy BDF-3299 at $z=7.1$. The goal of this work has been to 1) obtain a detailed map of the cold, clumpy gas structure by analysing a new [C II] observations at a resolution significantly higher (a factor 3) than previous observations (Maiolino et al. 2015); 2) map the [O III] emission in this primeval galaxy in order to further constrain the nature of its nature. 
About $70 \%( \pm 20 \%)$ of the [C II] emission detected by Maiolino et al. (2015) is resolved out in the high-angular resolution images indicating that a significant fraction of the [C II] emission is extended and diffuse on scales larger than about $1 \mathrm{kpc}$. We have combined the old ALMA low resolution and the new ALMA high resolution data to obtain deeper images at intermediate angular resolution. The resulting data show that the [C II] emission has a complex, clumpy structure. Both diffuse emission and multi-clumpy structure of the of the cold gas traced by the $[\mathrm{C} \mathrm{II}]$ emission, in and around primeval galaxies, is expected by recent models of primeval galaxies (e.g. Vallini et al. 2013, 2015, 2017; Pallottini et al. 2015, 2017; Fiacconi et al. 2017; Katz et al. 2016).

The new combined datacube also confirms the offset of [C II] relative to the optical ( $Y$-band) counterpart, by about $0.7^{\prime \prime}$ (4 kpc), although marginal detection of [C II] is also found at the location of the optical counterpart.

Furthermore we have reported the detection of [O III] emission line, with a level of confidence of $7.3 \sigma$. The line is also offset relative to the optical counterpart, in the same direction as the [C II] emission. However, most of the [O III] emission does not overlap with the [C II] emission. Two additional [O III] line emitters are also identified in the ALMA field of view, within $20 \mathrm{kpc}$ of BDF-3299.

We have shortly reviewed the the velocity and position of far-IR line emission in high- $z$ systems reported in literature, and we have shown that offsets relative to the optical counterparts, spatially or in velocity, are relatively common. We find that the offset, either spatial or in velocity, does not correlate with the SFR. While in some cases these offsets may be ascribed to astrometric uncertainties and to IGM absorption of $\operatorname{Ly} \alpha$, in other cases the offset are certainly associated with physically distinct components traced by optical and far-IR emission.

By comparing our data with models we show that the observed [O III] emission cannot be excited by the UV radiation coming from BDF-3299. The [O III] emission must be excited by in-situ star formation, with a $S F R \sim 7 M_{\odot} \mathrm{yr}^{-1}$. The lack of $Y$-band (UV-rest frame) counterpart at the location of the $[\mathrm{O}$ III $]$ clump(s) can be explained in terms of some modest amount of dust extinction. We note that $[\mathrm{O}$ III $] 88 \mu \mathrm{m}$ can be a powerful tool to detect normal star-forming galaxies with ALMA at $z>7$, competitive with HST, with the additional advantage of being insensitive to dust extinction.

We note that the non-detection of [C II] at the location of the [O III] clumps is consistent with the lower limit on $[\mathrm{O} \mathrm{III}] /[\mathrm{C} \mathrm{II}]$ observed with ALMA in a similar high- $z$ galaxy at $z=7.2$, and with local low metallicity galaxies. However, the high values of $[\mathrm{O}$ III $] /[\mathrm{C} \mathrm{II}]$ can also be interpreted in terms of high ionisation parameter, as suggested by some models.

The origin of the [C II] emission is more uncertain. Diffuse gas, associated with either satellite (accreting) gas rich systems or gas expelled by the optical galaxies, excited by the UV radiation field emitted by BDF-3299, is a viable interpretation based on the comparison with models. However, the scenario in which the bulk of the [C II] clumpy emission is excited by in-situ star formation (at a level of $3 M_{\odot} \mathrm{yr}^{-1}$ ) cannot be excluded either. The faint/absent [O III] emission at the location of [C II] can be interpreted in terms of either moderately high metallicity or low ionisation parameter.

Overall, the observational properties can be interpreted as a primeval system in the process of being assembled, in which different emission lines trace different components, characterised by different metallicity or excitation (ionisation parameter) properties. In this system the observable UV light is associated with the least obscured region, either because recently accreted, hence with low metallicity, or because strong feedback has removed the bulk of dust and gas content; both scenarios would account for the weakness of far-IR line emission at the location of the optical image.

Similar properties are observed in the much more powerful SMG systems at high redshift. Our observations are revealing that similar complex structures and processes are occurring also in normal primeval galaxies, with modest SFR.

Deeper ALMA data, possibly targeting also other transitions, are needed to obtain more detailed description of the system and to discriminate between different viable scenarios. JWST will also greatly help in understanding the nature of these system by probing the stellar population at optical rest-frame wavelengths and various nebular optical lines.

Acknowledgements. This paper makes use of the following ALMA data: ADS/JAO.ALMA\#2012.1.00719.S, ADS/JAO.ALMA\#2012.A.00040.S and, ADS/JAO.ALMA\#2013.A.00433.S; which can be retrieved from the ALMA data archive: https://almascience.eso.org/ alma-data/archive. ALMA is a partnership of ESO (representing its member states), NSF (USA) and NINS (Japan), together with NRC (Canada) and NSC and ASIAA (Taiwan), in cooperation with the Republic of Chile. The Joint ALMA Observatory is operated by ESO, AUI/NRAO and NAOJ. Based on observations made with the NASA/ ESA Hubble Space Telescope, obtained at the Space Telescope Science Institute, which is operated by the Association of Universities for Research in Astronomy, Inc., under NASA contract NAS 5-26555. These observations are associated with program \#13688. We thank Harley Katz and Martin Haehnelt for comments and discussions. S.C. acknowledges support by the Science and Technology Facilities Council (STFC). R.M. acknowledges support by the Science and Technology Facilities Council (STFC) and the ERC Advanced Grant 695671 "QUENCH”.

\section{References}

Amorín, R., Pérez-Montero, E., Contini, T., et al. 2015, A\&A, 578, A105 Aravena, M., Decarli, R., Walter, F., et al. 2016, ApJ, 833, 71

Bouwens, R. J., Illingworth, G. D., Oesch, P. A., et al. 2015, ApJ, 803, 34 Bouwens, R. J., Aravena, M., Decarli, R., et al. 2016, ApJ, 833, 72 Bradač, M., Garcia-Appadoo, D., Huang, K.-H., et al. 2017, ApJ, 836, L2 Bradley, L. D., Zitrin, A., Coe, D., et al. 2014, ApJ, 792, 76 Brauher, J. R., Dale, D. A., \& Helou, G. 2008, ApJS, 178, 280 Calvi, V., Trenti, M., Stiavelli, M., et al. 2016, ApJ, 817, 120 Calzetti, D., Armus, L., Bohlin, R. C., et al. 2000, ApJ, 533, 682 Capak, P. L., Carilli, C., Jones, G., et al. 2015, Nature, 522, 455 Carilli, C. L., Riechers, D., Walter, F., et al. 2013, ApJ, 763, 120 Carniani, S., Marconi, A., Biggs, A., et al. 2013, A\&A, 559, A29 Carniani, S., Maiolino, R., De Zotti, G., et al. 2015, A\&A, 584, A78 Castellano, M., Fontana, A., Paris, D., et al. 2010, A\&A, 524, A28 Castellano, M., Dayal, P., Pentericci, L., et al. 2016, ApJ, 818, L3 Cicone, C., Maiolino, R., Gallerani, S., et al. 2015, A\&A, 574, A14 Cormier, D., Lebouteiller, V., Madden, S. C., et al. 2012, A\&A, 548, A20 Dayal, P., Ferrara, A., Dunlop, J. S., \& Pacucci, F. 2014, MNRAS, 445, 2545 De Breuck, C., Williams, R. J., Swinbank, M., et al. 2014, A\&A, 565, A59 Decarli, R., Walter, F., Carilli, C., et al. 2014, ApJ, 782, L17 De Looze, I., Cormier, D., Lebouteiller, V., et al. 2014, A\&A, 568, A62 Dunlop, J. S., McLure, R. J., Biggs, A. D., et al. 2017, MNRAS, 466, 861 Erb, D. K., Steidel, C. C., Trainor, R. F., et al. 2014, ApJ, 795, 33 Federrath, C., \& Klessen, R. S. 2013, ApJ, 763, 51

Ferkinhoff, C., Hailey-Dunsheath, S., Nikola, T., et al. 2010, ApJ, 714, L147 Fiacconi, D., Mayer, L., Madau, P., et al. 2017, MNRAS, 467, 4080 Finkelstein, S. L., Papovich, C., Ryan, R. E., et al. 2012, ApJ, 758, 93 Finkelstein, S. L., Ryan, Jr., R. E., Papovich, C., et al. 2015, ApJ, 810, 71 Gallerani, S., Neri, R., Maiolino, R., et al. 2012, A\&A, 543, A114 Gallerani, S., Pallottini, A., Feruglio, C., et al. 2016, ArXiv e-prints [arXiv: 1604.05714] González-López, J., Riechers, D. A., Decarli, R., et al. 2014, ApJ, 784, 99 Graciá-Carpio, J., Sturm, E., Hailey-Dunsheath, S., et al. 2011, ApJ, 728, L7 Habing, H. J. 1968, Bull. Astron. Inst. Netherlands, 19, 421 Infante, L., Zheng, W., Laporte, N., et al. 2015, ApJ, 815, 18 Inoue, A. K., Shimizu, I., Tamura, Y., et al. 2014, ApJ, 780, L18 Inoue, A. K., Tamura, Y., Matsuo, H., et al. 2016, Science, 352, 1559 Ishigaki, M., Kawamata, R., Ouchi, M., et al. 2015, ApJ, 799, 12 
Kanekar, N., Wagg, J., Ram Chary, R., \& Carilli, C. L. 2013, ApJ, 771, L20

Katz, H., Kimm, T., Sijacki, D., \& Haehnelt, M. 2016, MNRAS, submitted [arXiv: 1612.01786$]$

Kaufman, M. J., Wolfire, M. G., Hollenbach, D. J., \& Luhman, M. L. 1999, ApJ, 527,795

Kawamata, R., Oguri, M., Ishigaki, M., Shimasaku, K., \& Ouchi, M. 2016, ApJ, 819,114

Kennicutt, R. C., \& Evans, N. J. 2012, ARA\&A, 50, 531

Knudsen, K. K., Richard, J., Kneib, J.-P., et al. 2016, MNRAS, 462, L6

Knudsen, K. K., Watson, D., Frayer, D., et al. 2017, MNRAS, 466, 138

Leitherer, C., Schaerer, D., Goldader, J. D., et al. 1999, ApJS, 123, 3

Madden, S. C., Poglitsch, A., Geis, N., Stacey, G. J., \& Townes, C. H. 1997, ApJ, 483, 200

Maiolino, R., Cox, P., Caselli, P., et al. 2005, A\&A, 440, L51

Maiolino, R., Caselli, P., Nagao, T., et al. 2009, A\&A, 500, L1

Maiolino, R., Gallerani, S., Neri, R., et al. 2012, MNRAS, 425, L66

Maiolino, R., Carniani, S., Fontana, A., et al. 2015, MNRAS, 452, 54

Malhotra, S., Kaufman, M. J., Hollenbach, D., et al. 2001, ApJ, 561, 766

Mallery, R. P., Mobasher, B., Capak, P., et al. 2012, ApJ, 760, 128

McLeod, D. J., McLure, R. J., Dunlop, J. S., et al. 2015, MNRAS, 450, 3032

McLure, R. J., Dunlop, J. S., Bowler, R. A. A., et al. 2013, MNRAS, 432, 2696

Negishi, T., Onaka, T., Chan, K.-W., \& Roellig, T. L. 2001, A\&A, 375, 566

Oesch, P. A., Bouwens, R. J., Illingworth, G. D., et al. 2014, ApJ, 786, 108

Oesch, P. A., Bouwens, R. J., Illingworth, G. D., et al. 2015, ApJ, 808, 104

Olsen, K. P., Greve, T. R., Narayanan, D., et al. 2015, ApJ, 814, 76

Ota, K., Walter, F., Ohta, K., et al. 2014, ApJ, 792, 34

Ouchi, M., Ellis, R., Ono, Y., et al. 2013, ApJ, 778, 102

Pallottini, A., Gallerani, S., Ferrara, A., et al. 2015, MNRAS, 453, 1898

Pallottini, A., Ferrara, A., Gallerani, S., et al. 2017, MNRAS, 465, 2540
Pavesi, R., Riechers, D. A., Capak, P. L., et al. 2016, ApJ, 832, 151

Pentericci, L., Carniani, S., Castellano, M., et al. 2016, ApJ, 829, L11 Pettini, M., Rix, S. A., Steidel, C. C., et al. 2002, ApJ, 569, 742

Pineda, J. L., Langer, W. D., \& Goldsmith, P. F. 2014, A\&A, 570, A121

Planck Collaboration XVI. 2014, A\&A, 571, A16

Rawle, T. D., Egami, E., Bussmann, R. S., et al. 2014, ApJ, 783, 59

Riechers, D. A., Carilli, C. L., Capak, P. L., et al. 2014, ApJ, 796, 84

Robertson, B. E., Ellis, R. S., Furlanetto, S. R., \& Dunlop, J. S. 2015, ApJ, 802, L19

Salvaterra, R., Ferrara, A., \& Dayal, P. 2011, MNRAS, 414, 847

Schaerer, D., Boone, F., Zamojski, M., et al. 2015, A\&A, 574, A19

Schmidt, K. B., Treu, T., Trenti, M., et al. 2014, ApJ, 786, 57

Shapley, A. E., Steidel, C. C., Pettini, M., \& Adelberger, K. L. 2003, ApJ, 588, 65

Stark, D. P., Ellis, R. S., Charlot, S., et al. 2017, MNRAS, 464, 469

Vallini, L., Gallerani, S., Ferrara, A., \& Baek, S. 2013, MNRAS, 433, 1567

Vallini, L., Gallerani, S., Ferrara, A., Pallottini, A., \& Yue, B. 2015, ApJ, 813, 36

Vallini, L., Ferrara, A., Pallottini, A., \& Gallerani, S. 2017, MNRAS, 467, 1300

Vanzella, E., Pentericci, L., Fontana, A., et al. 2011, ApJ, 730, L35

Venemans, B. P., McMahon, R. G., Walter, F., et al. 2012, ApJ, 751, L25

Wagg, J., Wiklind, T., Carilli, C. L., et al. 2012, ApJ, 752, L30

Walter, F., Riechers, D., Cox, P., et al. 2009, Nature, 457, 699

Walter, F., Decarli, R., Carilli, C., et al. 2012, ApJ, 752, 93

Walter, F., Decarli, R., Aravena, M., et al. 2016, ApJ, 833, 67

Williams, R. J., Wagg, J., Maiolino, R., et al. 2014, MNRAS, 439, 2096

Willott, C. J., Carilli, C. L., Wagg, J., \& Wang, R. 2015, ApJ, 807, 180

Wolfire, M. G., McKee, C. F., Hollenbach, D., \& Tielens, A. G. G. M. 2003, ApJ, 587,278

Zitrin, A., Zheng, W., Broadhurst, T., et al. 2014, ApJ, 793, L12 


\section{Appendix A: Extended [C II] emission}

In this Appendix, we compare the [C II] emission detected in the ALMA Cycle 1 and Cycle 2 and we discuss the differences between the outcomes of these projects.

The Top panel of Fig. A.1 shows the Cycle 2 ALMA spectrum extracted at the location of the [C II] detection $(\operatorname{RA}(J 2000)=22: 28: 12.325, \operatorname{Dec}(J 2000)=-35: 10: 00.64)$ and from a elliptical aperture of $0.55^{\prime \prime} \times 1.03^{\prime \prime}$ (approx. nine beams) corresponding to the extension of the clump inferred by Maiolino et al. (2015). The carbon line is not detectable in the integrated line because of the low sensitivity in the high-spatial resolution observations. In fact, the noise level $(0.35 \mathrm{mJy}$ in element channels of $40 \mathrm{~km} \mathrm{~s}^{-1}$ ) in the integrated spectra is comparable with the peak of [C II] line $(0.39 \mathrm{mJy})$. The highresolution integrated map (bottom panel of Fig. A.1), whose sensitivity is 1.4 times lower than the integrated map obtained with the compact ALMA configuration, does not reveal any highsignificance extended or multi-clumpy emission, suggesting that the $[\mathrm{C} \mathrm{II}]$ emission is mostly resolved out in the ALMA extended configuration, although there a number of $1 \sigma$ and $2 \sigma$ emissions spots that will contribute to the signal in the total, combined data set. In fact, if the emission was powered by a compact source unresolved at this resolution, we would expect to observe the peak of the emission with a level of confidence of $\sim 3-3.5$ in the integrated map.
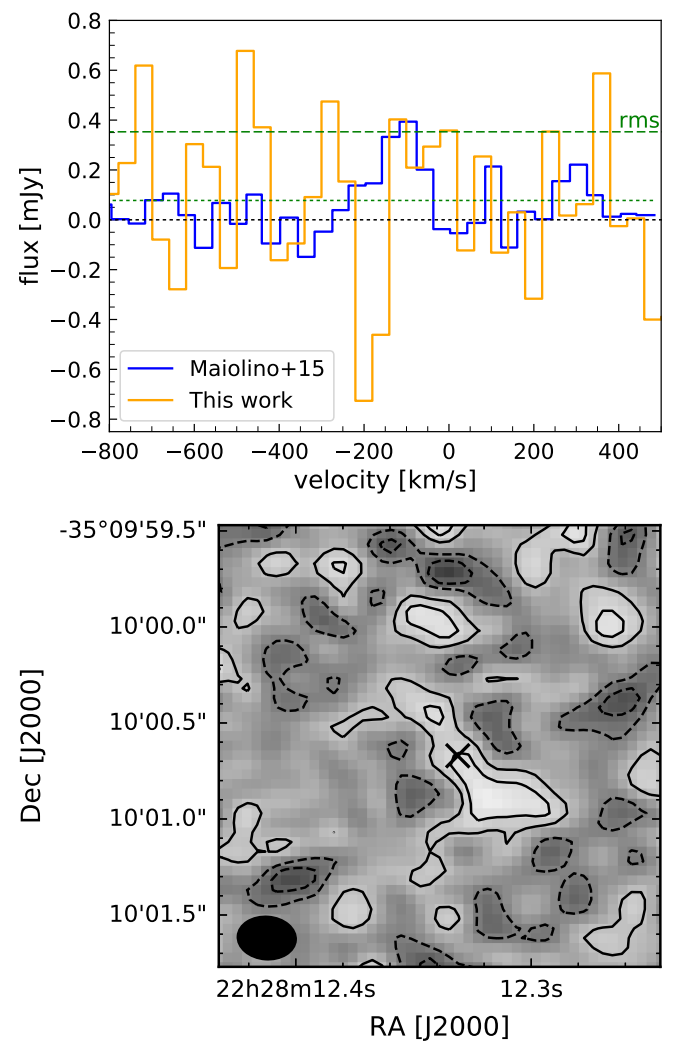

Fig. A.1. Top: ALMA spectrum of the [C II] detections, extracted from the an elliptical aperture of size $0.55^{\prime \prime} \times 1.03^{\prime \prime}$ with resolution of $40 \mathrm{~km} \mathrm{~s}^{-1}$. The blue solid line is the spectrum by Maiolino et al. (2015), while the orange and green lines show the spectrum and noise level obtained from the new high-resolution observations, respectively. Bottom: high-resolution ALMA map (angular-resolution $\left.=0.3^{\prime \prime} \times 0.2^{\prime \prime}\right)$ obtained by integrating the line under the gold shaded region in the top panel of Fig. 2 a (i.e. $-70<v<10 \mathrm{~km} \mathrm{~s}^{-1}$ ). Black contours are at levels of $-2,-1,1$, and 2 times the noise per beam in the same map, i.e. $9 \mathrm{mJy} / \mathrm{beam} \mathrm{km} \mathrm{s}^{-1}$. 